\title{
Effectiveness of a multidisciplinary comprehensive intervention model based on the Hospital Elderly Life Program to prevent delirium in patients with severe acute pancreatitis
}

\author{
Zhixia Dong', Jie Song ${ }^{1}$, Minhua Ge ${ }^{1}$, Chunyan Lin ${ }^{1}$, Jie Zhang ${ }^{2}$, Jie Chen ${ }^{2}$, Yanmin Wu ${ }^{1}$ \\ ${ }^{1}$ Department of Gastroenterology, ${ }^{2}$ Department of Hepatobiliary, the Affiliated Hospital of Jiangnan University, Wuxi, China \\ Contributions: (I) Conception and design: Z Dong, J Song, M Ge, C Lin, Y Wu; (II) Administrative support: J Song; (III) Provision of study materials \\ or patients: Z Dong, M Ge, C Lin, J Zhang, J Chen, Y Wu; (IV) Collection and assembly of data: Z Dong, J Song, M Ge, C Lin, Y Wu; (V) Data \\ analysis and interpretation: Z Dong, C Lin, Y Wu; (VI) Manuscript writing: All authors; (VII) Final approval of manuscript: All authors. \\ Correspondence to: Yanmin Wu. Department of Gastroenterology, Affiliated Hospital of Jiangnan University, Wuxi, China. \\ Email: 396285432@qq.com.
}

Background: Pancreatic encephalopathy is the most serious complication of severe acute pancreatitis (SAP) and substantially increases mortality. Delirium is a common clinical manifestation of pancreatic encephalopathy. This study aimed to assess the clinical effects of an improved version of the Hospital Elderly Life Program (HELP) on preventing delirium in elderly patients with SAP.

Methods: A total of 106 patients with SAP aged 70 years or older were randomly divided into an experimental group (53 cases) and a control group (53 cases). The experimental group was given 2 weeks of conventional nursing combined with the HELP regimen, while the control group was given 2 weeks of conventional nursing. Conventional nursing included disease care, geriatric syndrome care and observation of critically ill patients. The incidence of delirium, severity of delirium, self-care ability, cognitive function, length of hospital stay, and patient satisfaction were compared between the 2 groups before and after the intervention.

Results: The incidence of delirium was $4.00 \%$ in the experimental group and $16.98 \%$ in the control group; the difference was statistically significant $(\mathrm{P}=0.033)$. After 2 weeks of intervention, the experimental group showed significantly better self-care ability and cognitive function, shorter hospital stay, and greater patient satisfaction than the control group (all $\mathrm{P}<0.05$ ).

Conclusions: The improved HELP protocol for elderly patients with SAP can reduce the incidence and severity of delirium, improve self-care ability and cognitive function, shorten hospital stay, and increase patient satisfaction.

Keywords: Pancreatitis; aged; delirium; geriatric nursing; cognitive function; self-care

Submitted Mar 19, 2020. Accepted for publication Jul 15, 2020.

doi: 10.21037/apm-20-913

View this article at: http://dx.doi.org/10.21037/apm-20-913

\section{Introduction}

Severe acute pancreatitis (SAP) is one of the most common acute, severe abdominal diseases. Its clinical manifestation, disease course, and prognosis are complex and changeable (1). At present, the commonly used nursing methods of SAP in China mainly include conventional nursing, cluster nursing and quality nursing. But the results of these nursing methods are not satisfactory. Pancreatic encephalopathy is the most serious complication of SAP. The mortality of patients with severe pancreatitis who develop pancreatic encephalopathy increases to more than 70\% (2). Delirium is one of the most common clinical manifestations of pancreatic encephalopathy. This is an acute, reversible, fluctuating state of brain dysfunction characterized by 
attention problems, altered consciousness, disorientation, and disordered cognition, thought, and sleep. Delirium is particularly prevalent in elderly people (3), and can lead to decreased self-care ability, prolonged hospital stay, and increased readmission rates in elderly hospitalized patients (4-6). Therefore, it is important to reduce the incidence and harmful effects of delirium. The current domestic and foreign evidence and related guidelines (7) do not recommend the sole use of drugs to prevent delirium; rather, non-drug multidisciplinary and multifactorial comprehensive intervention programs are strongly recommended to prevent delirium in elderly patients. The Hospital Elderly Life Program (HELP) is a hospitalbased, patient-centered, nurse-led, National Institute for Health and Care Excellence (NICE) guideline-based, nondrug delirium prevention model (8). The HELP has been tested in more than 200 medical institutions worldwide, and evidence shows that it can prevent delirium and physical or cognitive decline in elderly patients in hospital. However, the HELP has not yet been used in delirium in elderly patients with SAP, so this study developed an improved HELP protocol and aimed to evaluate the effectiveness of the improved HELP in preventing delirium in elderly patients with SAP using a clinical randomized controlled trial. It is hoped that our findings will provide evidence for the further promotion and application of this model. We present the following article in accordance with the CONSORT Statement for Randomized Trials of Nonpharmacologic Treatments reporting checklist (available at http://dx.doi.org/10.21037/apm-20-913).

\section{Methods}

\section{Subjects}

Subjects were 106 patients with SAP admitted to the Affiliated Hospital of Jiangnan University, China, from December 2016 to December 2019. Using the random number table method, subjects were randomly divided into a test group (53 cases) and a control group (53 cases). The inclusion criteria were the following: (I) meeting the SAP diagnostic criteria in the 2013 Chinese Guidelines for the Diagnosis and Treatment of Acute Pancreatitis (9); (II) aged 70 years or older; (III) expected hospital stay >2 weeks; and (IV) provision of written informed consent. The exclusion criteria were the following: (I) history of SAP; (II) coma; (III) complicated with mental disorders or disorders; (IV) dementia; (V) low immune function (such as neutrophil deficiency); and (VI) end-stage disease. This study was approved by the ethics committee of the Affiliated Hospital of Jiangnan University [(2016)KY039] and was performed in accordance with the Helsinki Declaration (revised in 2013). All subjects agreed and signed informed consent.

\section{Interventions}

(I) Intervention for the experimental group consisted of routine nursing combined with the modified HELP intervention used from the 1st day of admission to the 14th day of admission. If the patient was discharged within 14 days, the intervention was continued until the day of discharge. (II) Intervention for the control group consisted of use of routine nursing programs and procedures. Details of the interventions are shown in Table 1.

\section{Evaluation indexes}

\section{Incidence of delirium}

The 3D-Confusion Assessment Method scale (CAMS) was used to evaluate delirium incidence. This assesses 4 basic characteristics: (I) acute attacks and volatile changes; (II) inattention; (III) confusion of thinking; and (IV) changes in consciousness level. The scale has high sensitivity (94$100 \%$ ) and specificity (90-95\%) (9). Delirium is confirmed if the patient has the following combination of the above items: 1 and 2 plus 3 , or 1 and 2 plus 4 .

\section{Severity of delirium}

The full version of CAMS and the Memory Delirium Assessment Scale (MDAS) were used to score delirium severity (10). Both comprise 10 items. CAM-S items are rated from 0 to 19 and MDAS items are rated from 0 to 30; higher scores indicate more severe delirium.

\section{Changes in cognitive function}

The Short Portable Mental Status Questionnaire (SPMSQ) (11) was used to assess cognitive function. The questionnaire comprises 10 items, and each item contains 4 dimensions: orientation, personal history, memory (longterm, short-term memory), and computational power. Scores were categorized as intact mental function, mild intellectual impairment, moderate intellectual impairment, and severe intellectual impairment.

\section{Self-care ability}

The Barthel Index (BI) (12) was used to evaluate activities 
Table 1 Comparison of intervention programs in the experimental group and the control group

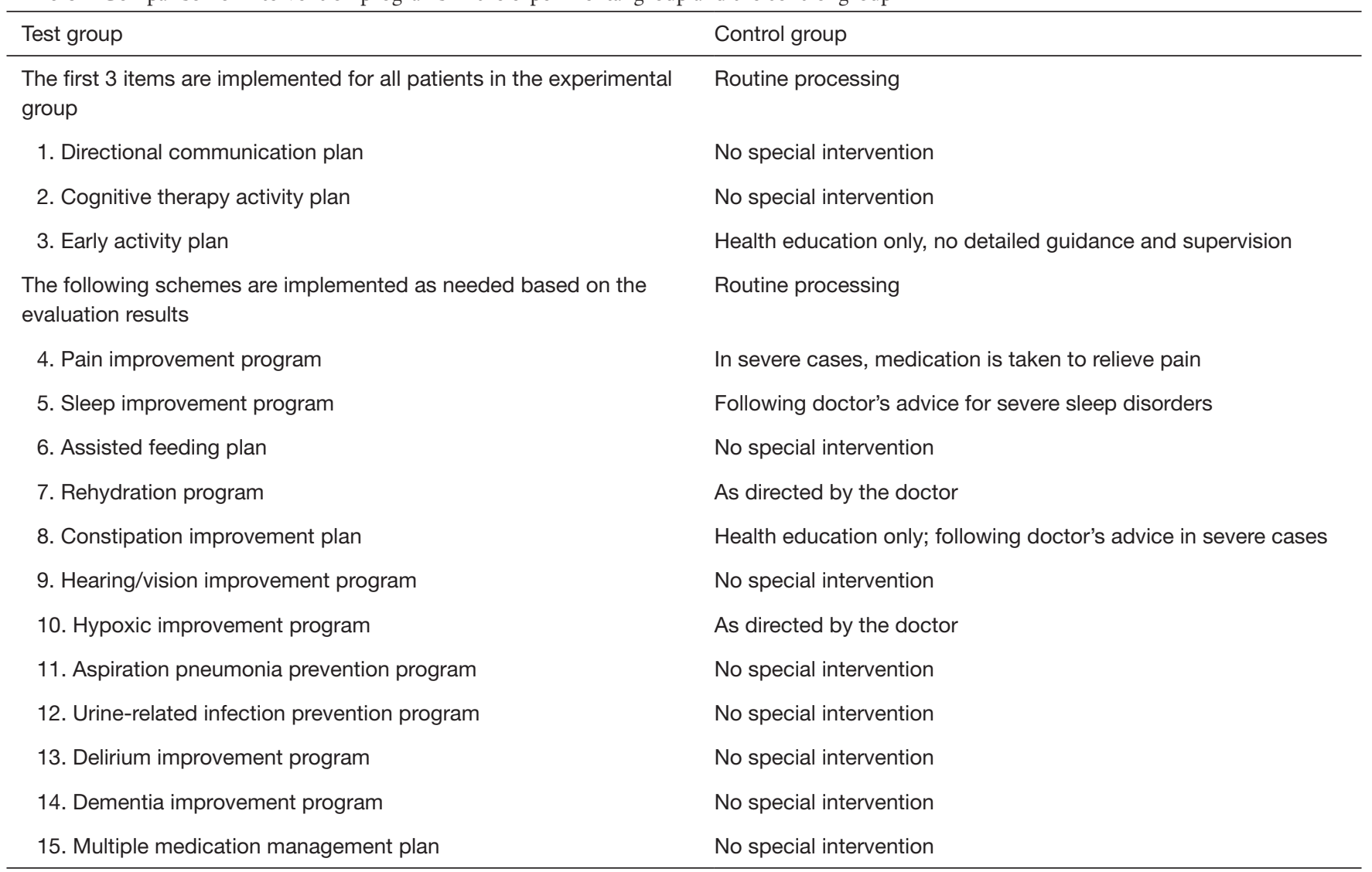

of daily living. The BI comprises 10 items that assess eating, bathing, grooming, dressing, using the toilet, bed and chair transfer, activities, going up and down stairs, controlling stool, and controlling urination. The total possible score is 100; higher scores indicate greater independence. The total score is categorized as follows: $100=$ normal ability to perform activities of daily living, 61-99 basic self-care ability (mild dependence), 41-60 self-care ability (moderate dependence), $0-40$ no self-care ability (heavy dependence).

\section{Patient satisfaction}

On the basis of literature review and consulting experts, we prepared our own questionnaire on satisfaction with delirium in elderly patients with SAP. Questionnaire mainly included medical circumstances (10 points), incomplete process (15 points), the technology level of doctors (15 points), the technology level of nurses (15 points), the service attitude of doctors (15 points), the service attitude of nurses (15 points) and complications (15 points), with a score of 100 points, the higher the score, the better the satisfaction.

\section{Statistical analysis}

SPSS 22.0 (SPSS Inc., Chicago, IL, USA) was used for statistical analysis. Measurement data were described as mean \pm standard deviation, and compared using 2 independent sample $t$-tests. Count data were described using rates and percentages, and compared using the $\chi^{2}$ test or rank-sum test. Values of $\mathrm{P}<0.05$ were considered to indicate statistically significant differences.

\section{Results}

\section{Baseline characteristics of patients}

There were 53 patients in the experimental group; 3 of these patients died and the remaining 50 patients completed the study. The 53 patients in the control group completed the study. There was no significant difference in baseline 
data between the 2 groups $(\mathrm{P}>0.05)$, as shown in Table 2 .

\section{Incidence of delirium}

In the test group, 2 patients had delirium during hospitalization (a delirium incidence of $4.00 \%$ ), while in the control group, 9 patients had delirium (a delirium incidence of $16.98 \%)$. The difference in the incidence of delirium between the 2 groups was statistically significant $\left(\chi^{2}=4.545\right.$, $\mathrm{P}=0.033)$.

\section{Severity of delirium}

The severity of delirium was significantly lower in the experimental group than in the control group. Betweengroup differences in full CAM-S score $(\mathrm{t}=2.555, \mathrm{P}=0.012)$ and MDAS score $(\mathrm{t}=4.2869, \mathrm{P}=0.000)$ were statistically significant, as shown in Table 3.

\section{Cognitive function}

At the time of admission, there was no significant difference in cognitive function between the experimental group and the control group $(\mathrm{Z}=0.159, \mathrm{P}=0.924)$. After 2 weeks of intervention, the cognitive function of patients in the experimental group improved compared with baseline. The post-intervention difference between the experimental group and the control group was statistically significant $(\mathrm{Z}=8.823, \mathrm{P}=0.12)$, as shown in Table 4 .

\section{Self-care ability}

At the time of admission, there was no significant difference in cognitive function between the experimental group and the control group $(\mathrm{Z}=1.491, \mathrm{P}=0.684)$. After 2 weeks of intervention, the self-care ability of patients in the experimental group improved compared with baseline. The post-intervention difference between the experimental group and the control group was statistically significant $(\mathrm{Z}=11.363, \mathrm{P}=0.010)$, as shown in Table 5 .

\section{Hospital stay and patient satisfaction}

Compared with the control group, the average hospital stay was significantly lower in the experimental group, and the difference was statistically significant $(\mathrm{t}=8.329, \mathrm{P}=0.000)$. Patient satisfaction score was significantly higher in the experimental group, and the difference was statistically significant $(\mathrm{t}=8.764, \mathrm{P}=0.000)$, as shown in Table 6 .

\section{Discussion}

The occurrence of delirium is the result of a combination of factors (13). The presence of more risk factors increases the risk of delirium (14). Prevention of delirium should be based on its risk factors and a comprehensive multidisciplinary non-drug intervention program (15). Delirium care for patients with conventional SAP is managed by nurses alone and lacks comprehensiveness. The HELP is a patientcentered, multidisciplinary, collaborative, comprehensive non-drug intervention for delirium risk factors, which can compensate for the limitations of routine care. The effects of the HELP have been confirmed by multiple studies. This study revised the HELP using relevant expert opinion from medical and health professionals. Compared with the conventional HELP, the improved HELP had the following contents: delirium improvement plan, dementia improvement plan and multiple medication management plan. The improved HELP enables the assessment of delirium risk factors, delirium diagnosis, and multidisciplinary intervention for elderly patients with SAP. The present findings show that the improved HELP protocol can reduce the incidence and severity of delirium in elderly patients with SAP. However, previous studies from a range of countries show conflicting findings regarding the value of the HELP intervention. Inouye et al. (14) proposed that the HELP intervention has no clear effect on improving the severity of delirium, whereas Marcantonio et al. (16) reported that it can substantially improve delirium severity. The evaluation of delirium severity is difficult, so the present study used 2 delirium severity scales to improve the reliability of the results.

The present findings indicate that the improved HELP protocol can improve self-care ability and cognitive function in patients with SAP. Impaired self-care ability and cognitive dysfunction in elderly people are closely related, and both are also risk factors for delirium (17). Cognitive function intervention is an important aspect of the improved HELP. Through daily targeted communication, such intervention can effectively prevent and improve patient cognitive function, and thus prevent delirium. This result is consistent with the research results of Reuben et al. (18). Early intervention to improve patient self-care is also an important aspect of the improved HELP. Early implementation of purposeful and planned activity interventions can maintain physical function, improve self-care ability, and reduce 
Table 2 Comparison of baseline characteristics in the two patient groups (n, \%)

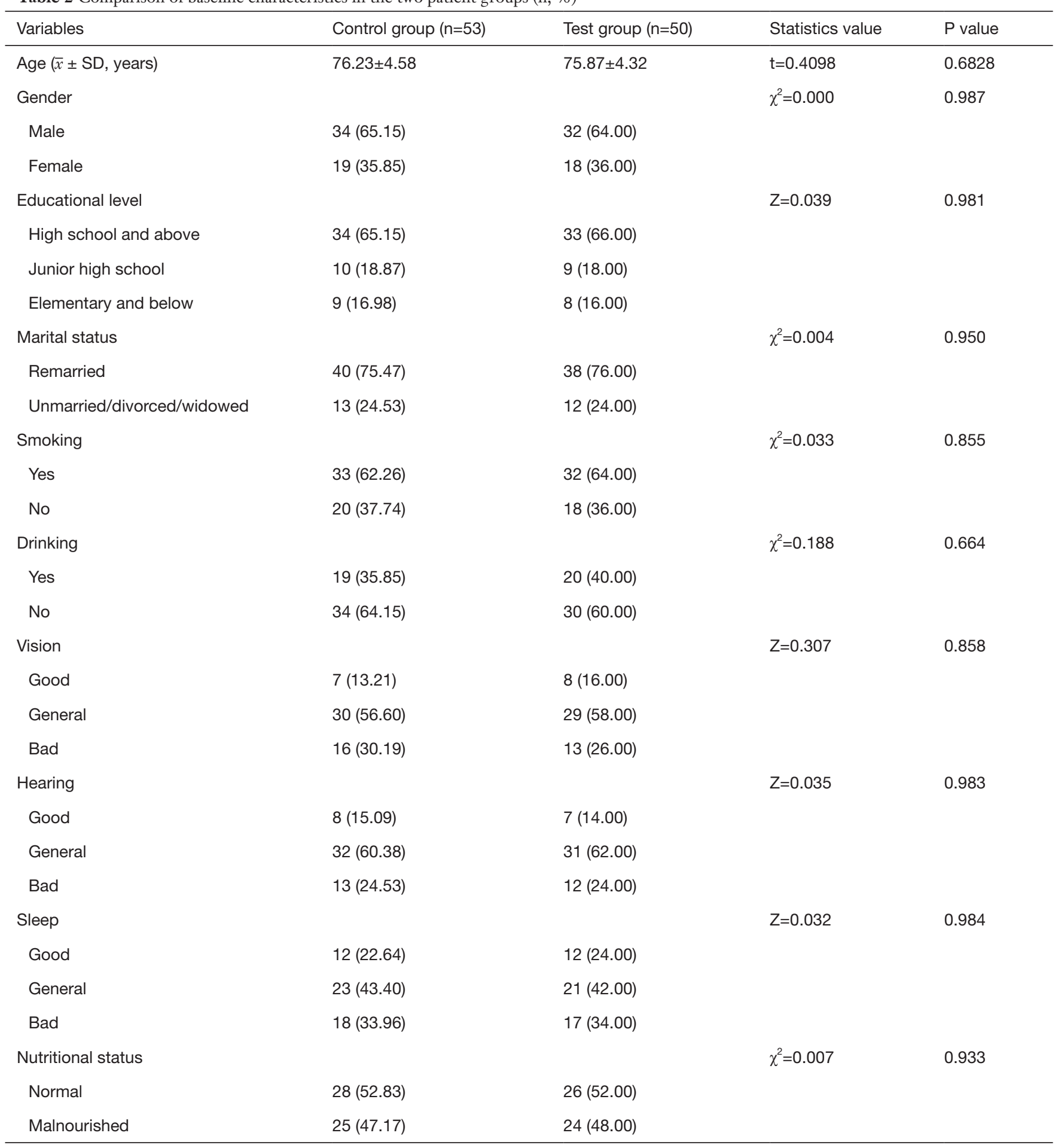


Table 3 Comparison of delirium severity in the two patient groups (n, $\bar{x} \pm \mathrm{SD})$

\begin{tabular}{|c|c|c|c|c|}
\hline Variables & Control group $(n=53)$ & Test group $(n=50)$ & Statistics value & $P$ value \\
\hline MDAS score & $24.12 \pm 2.76$ & $21.84 \pm 2.63$ & $t=4.287$ & 0.000 \\
\hline
\end{tabular}

CAM, Confusion Assessment Method; MDAS, Memory Delirium Assessment Scale.

Table 4 Comparison of cognitive function before and after intervention in the two patient groups (n, \%)

\begin{tabular}{|c|c|c|c|c|}
\hline Time & Control group $(n=53)$ & Test group $(n=50)$ & Statistics value & $P$ value \\
\hline Intact mental function & $35(66.04)$ & $32(64.00)$ & & \\
\hline Mild intellectual impairment & $9(16.98)$ & $10(20.00)$ & & \\
\hline Moderate and severe intellectual impairment & $9(16.98)$ & $8(16.00)$ & & \\
\hline Intact mental function & $32(60.38)$ & $37(74.00)$ & & \\
\hline Mild intellectual impairment & $8(15.09)$ & $11(22.00)$ & & \\
\hline Moderate and severe intellectual impairment & $13(24.53)$ & $2(4.00)$ & & \\
\hline
\end{tabular}

Table 5 Comparison of self-care ability before and after intervention in the two patient groups (n, \%)

\begin{tabular}{|c|c|c|c|c|}
\hline Time & Control group $(n=53)$ & Test group $(n=50)$ & Statistics value & $P$ value \\
\hline Self-care ability & $9(16.98)$ & $5(10.00)$ & & \\
\hline Mild dependence & $28(52.83)$ & $26(52.00)$ & & \\
\hline Moderate dependence & $11(20.75)$ & $14(28.00)$ & & \\
\hline 2 weeks after intervention & & & $Z=11.363$ & 0.010 \\
\hline Self-care ability & $5(9.43)$ & $11(22.00)$ & & \\
\hline Mild dependence & $21(39.62)$ & $28(56.00)$ & & \\
\hline Moderate dependence & $16(30.19)$ & $9(18.00)$ & & \\
\hline
\end{tabular}

Table 6 Comparison of hospitalization time and patient satisfaction in the two patient groups $(\bar{x} \pm$ SD)

\begin{tabular}{lccc}
\hline Variables & Control group $(n=53)$ & Test group $(n=50)$ & Statistics value \\
\hline Hospital stay (days) & $16.03 \pm 2.45$ & $12.26 \pm 2.12$ & $t=8.329$ \\
Satisfaction (points) & $84.75 \pm 5.69$ & $95.08 \pm 6.27$ & $t=8.764$ \\
\hline
\end{tabular}


the incidence of delirium in elderly people. This result is consistent with the results of previous studies $(8,19)$.

This study also found that the modified HELP protocol can shorten the length of hospital stay in elderly patients with SAP. However, Chen et al. (20) found that the HELP regimen did not significantly shorten the length of hospital stay. This may be related to regional differences in hospital management systems, disease severity, and discharge standards. Therefore, an in-depth analysis of other influencing factors is needed. The present study also evaluated patient satisfaction with medical care during hospitalization. The results show that the improved HELP increased patient satisfaction, was accepted by patients, and could be applied to current and future elderly medical care models in China.

Based on the time limitation, the sample size of this study is relatively small, the observation items are relatively limited, and there is a lack of in-depth and accurate laboratory indicators for the measurement. It is expected that there will be further studies on the efficacy of deeper efficacy HELP in the treatment of delirium in elderly patients with SAP in the future, so as to form a more objective and accurate conclusion.

\section{Conclusions}

The modified HELP targets the risk factors of delirium and provides individualized multidisciplinary non-drug interventions for elderly patients with SAP. The findings of this study indicate that the program can effectively reduce the incidence and severity of delirium, shorten the length of hospital stay, strengthen self-care ability and cognitive function, and improve patient satisfaction.

\section{Acknowledgments}

We thank this fund and the Affiliated Hospital of Jiangnan University for providing experimental equipment support for the study. We thank Diane Williams, PhD, from Liwen Bianji, Edanz Editing China (www.liwenbianji.cn/ac), for editing the English text of a draft of this manuscript.

Funding: This work was supported by the Translational Medicine Specialty of Wuxi Municipal Health Committee (ZM006).

\section{Footnote}

Reporting Checklist: The authors have completed the
CONSORT Statement for Randomized Trials of Nonpharmacologic Treatments reporting checklist. Available at http://dx.doi.org/10.21037/apm-20-913

Data Sharing Statement: Available at http://dx.doi. org/10.21037/apm-20-913

Conflicts of Interest: All authors have completed the ICMJE uniform disclosure form (available at http://dx.doi. org/10.21037/apm-20-913). The authors have no conflicts of interest to declare.

Ethical Statement: The authors are accountable for all aspects of the work in ensuring that questions related to the accuracy or integrity of any part of the work are appropriately investigated and resolved. This study was performed in compliance with the ethical principles of the Declaration of Helsinki (as revised in 2013) and was approved by the Ethics Committee of the Affiliated Hospital of Jiangnan University [(2016)KY039]. All subjects agreed and signed informed consent.

Open Access Statement: This is an Open Access article distributed in accordance with the Creative Commons Attribution-NonCommercial-NoDerivs 4.0 International License (CC BY-NC-ND 4.0), which permits the noncommercial replication and distribution of the article with the strict proviso that no changes or edits are made and the original work is properly cited (including links to both the formal publication through the relevant DOI and the license). See: https://creativecommons.org/licenses/by-nc-nd/4.0/.

\section{References}

1. Hallac A, Puri N, Applebury D, et al. The value of quick sepsis-related organ failure assessment scores in patients with acute pancreatitis who present to emergency departments: a three-year cohort study. Gastroenterology Res 2019;12:67-71.

2. Mao E. Intensive management of severe acute pancreatitis. Ann Transl Med 2019;7:687.

3. Hess S, Zemishlany Z. The psychiatric diagnosis guideDSM-5-nnovations and criticism. Harefuah 2015;154:31922, 38.

4. Pendlebury ST, Lovett NG, Smith SC, et al. Observational, longitudinal study of delirium in consecutive unselected acute medical admissions: agespecific rates and associated factors, mortality and re- 
admission. BMJ Open 2015;5:e007808.

5. Inouye SK, Marcantonio ER, Kosar CM, et al. The shortterm and long-term relationship between delirium and cognitive trajectory in older surgical patients. Alzheimers Dement 2016;12:766-75.

6. Tropea J, Logiudice D, Liew D, et al. Poorer outcomes and greater healthcare costs for hospitalised older people with dementia and delirium: a retrospective cohort study. Int J Geriatr Psychiatry 2017;32:539-47.

7. Barr J, Fraser GL, Puntillo K, et al. Clinical practice guidelines for the management of pain, agitation, and delirium in adult patients in the Intensive Care Unit I executive summary. Am J Health Syst Pharm 2013;70:53-8.

8. Inouye SK, Jr BS, Baker DI, et al. The Hospital Elder Life Program: a model of care to prevent cognitive and functional decline in older hospitalized patients. Hospital Elder Life Program. J Am Geriatr Soc 2000;48:1697.

9. Inouye SK, Kosar CM, Tommet D, et al. The CAM-S development and validation of a new scoring system for delirium severity in 2 cohorts. Ann Intern Med 2014;160:526-33.

10. Fadul N, Kaur G, Zhang T, et al. Evaluation of the memorial delirium assessment scale (MDAS) for the screening of delirium by means of simulated cases by palliative care health professionals. Support Care Cancer 2007;15:1271-6.

11. Pfeiffer E. A short portable mental status questionnaire for the assessment of organic brain deficit in elderly patients. J Am Geriatr Soc 1975;23:433-41.

Cite this article as: Dong Z, Song J, Ge M, Lin C, Zhang J, Chen J, Wu Y. Effectiveness of a multidisciplinary comprehensive intervention model based on the Hospital Elderly Life Program to prevent delirium in patients with severe acute pancreatitis. Ann Palliat Med 2020;9(4):2221-2228. doi: 10.21037/apm-20-913
12. Mahoney FI, Barthel DW. Functional evolution: the Barthel Index. Md State Med J 1965;14:61-5.

13. Feng Q, Li L, Ma W, et al. Analysis of occurrence and influencing factors of delirium in patients with terminal cancer. Chinese Journal of Nursing 2019;54:238-43.

14. Inouye SK, Bogardus ST Jr, Charpentier PA, et al. A multicom-ponent intervention to prevent delirium in hospitalized older patients. N Engl J Med 1999;340:669-76.

15. Yue J, Tabloski P, Dowal SL, et al. NICE to HELP: operationalizing National Institute for Health and Clinical Excellence guidelines to improve clinical practice. J Am Geriatr Soc 2014;62:754-61.

16. Marcantonio ER, Flacker JM, Wright RJ, et al. Reducing delirium after hip fracture: a randomized trial. J Am Geriatr Soc 2001;49:516-22.

17. Tam CW, Lam LC. Cognitive and functional impairment in Chinese elderly with late-onset depression. East Asian Arch Psychiatry 2012;22:25-30.

18. Reuben DB, Sharon K, Inouye MD, et al. Models of geriatrics practice; the prevent cognitive and functional decline in older hospitalized patients. J Am Geriatr So 2000;48:1697-706.

19. Li MM, Luo J, Xie L, et al. A literature review of ABCDEF bundle to prevent ICU-acquired delirium and ICU-acquired weakness. Chinese Journal of Nursing 2018;53:358-63.

20. Chen CC, Lin MT, Tien YW, et al. Modified hospital elder life program I effects on abdominal surgery patients. J Am Coll Surg 2011;213:245-52. 